\title{
ET-OLSR: Energy based Threshold Optimized Link State Routing for MANETs
}

\author{
Navdeep Kaur \\ University Institute of Engineering and \\ Technology, Panjab University \\ Chandigarh [INDIA]
}

\author{
Inderdeep Aulakh \\ University Institute of Engineering and \\ Technology, Panjab University \\ Chandigarh [INDIA]
}

\begin{abstract}
Mobile Ad-hoc Networks (MANETs) are the one where each node participating has many roles assigned, that is of a receiver, transmitter and router. Hence, energy in abundance is expected to be expended on each role to achieve the normal working. Also, mobility factor of this infrastructure-less administration forces the nodes to confide on battery energy. Battery being a limited entity fails to fulfill the high demand of energy with time. Scarcity of battery life and the additional energy requirements for supporting network operations (e.g. routing) inside every node, make the energy conservation one of the peer concern in ad hoc networking. Significant research effort has been made on efficient use of energy. Several exemplary techniques include transmission power control, load distribution, power management and sleep mode approach. This paper takes up a new optimized energy consumption algorithm emanated from power management technique to be put into effect on Optimized Link State Routing (OLSR) protocol. The procedure follows a threshold value for each node that indicates whether the node should participate in path discovery for a packet. Eventually, the contrast behavior of OLSR is examined. For that, extensive simulations have been carried with a network setup, such that the nodes less than this specified threshold value get eliminated from route. Comparison graphs plotted for new Energy Threshold OLSR (ET-OLSR) and OLSR conclude that performance of new protocol outshines in terms of energy consumption and packet delivery ratio.
\end{abstract}

\section{General Terms}

MANET, Mobile Ad hoc Networks, Energy Efficiency

\section{Keywords}

OLSR, Energy threshold, Energy consumption, Energy conservation, Power consumption, Energy aware algorithms

\section{INTRODUCTION}

With the emergence of low cost wireless networking, wide range of applications are been incorporated in laptops, embedded systems and cellular phones. To enable such availability Mobile Ad-hoc Network (MANET) play a vital role, as it provides communication without any fixed infrastructure. Environment created by MANETs [1] [2] [3] emerges as a miscellany of wireless nodes which forms a network for a short span without any assistance of centralized infrastructure.

The routing in such architecture is bifurcated as reactive (on demand) and proactive (table driven) [4]. Reactive routing protocols are featured by a path discovery mechanism that is triggered on demand, when an information unit needs to get to a given destination. Whereas, proactive routing maintains route tables that store the routing information with any change in the network topology and trigger propagating updates throughout the network in order to maintain a consistent network view.

With inevitable evolution in communication and network technologies, we have experienced many potential gains. Simultaneously, the complex nature of the existing networked information system has already overpowered the conventional networking paradigms in context of deploying, managing, and maintaining them correctly. Evidence is the energy dilemma in MANETs [5] [6].

As the scenario is not fixed, nodes rely upon battery, which depletes with time. This arise the crunch of energy, as a constraint in MANETs, which calls for efficient energy utilization. Several exemplary techniques have been taken up to minimize active as well as inactive communication energy. In these schemes, many algorithms [7] emerged for both reactive and proactive routing in MANETs, which contributed for energy efficiency to a large extent. In 2012, Suganya and Palaniammal proposed an algorithm [8], where threshold value was implemented over each node for energy conservation. The node having energy less than the threshold got destroyed from the path to be traversed and optimum path was followed.

Generally, for the statistical analysis of energy conservation and consumption in traffic models (CBR, Pareto and Exponential) routing protocols used are AODV, TORA, OLSR, AOMDV, DSDV and DSR. This paper has considered the recently introduced algorithm mentioned before [8] to be implemented over OLSR protocol.

OLSR has outperformed its conventional link state protocols as it is optimized by introducing Multipoint Relays (MPRs). MPRs are nodes that are selected for forward broadcast messages during the flooding process according to RFC 3626[9]. OLSR [10] being proactive in nature has the ability to find routes between two nodes in a network in less time, also it is said to be already aware of energy as a limitation. This paper is framed as follows: Section II covers the relevant work already done for enhancing the energy in networks. It confers on related algorithms that are been used till now. Further, Section III presents the actual simulation scenario along with the description of algorithm used. Section IV exhibits the simulation results obtained in NS2 environment. Finally, conclusions and future work are derived in the last part of the paper.

\section{ENERGY AWARE ALGORITHMS}

The growing need for energy efficiency in wireless networks, in general, and in mobile ad hoc networks, in particular, calls for power enhancement features. There are active communication energy techniques [11] that can be categorized as Transmission Power Control, Load distribution and Power management and inactive communication energy minimizing takes up the sleep mode approach [12]. Apart 
from this, Natural engineering [13] approach has also contributed with its nature gifted ways to conserve energy for energy depreciating environments like MANETs. Figure 1 exhibits the clear classification of techniques used, also a detailed illustration of each approach is given below with examples.

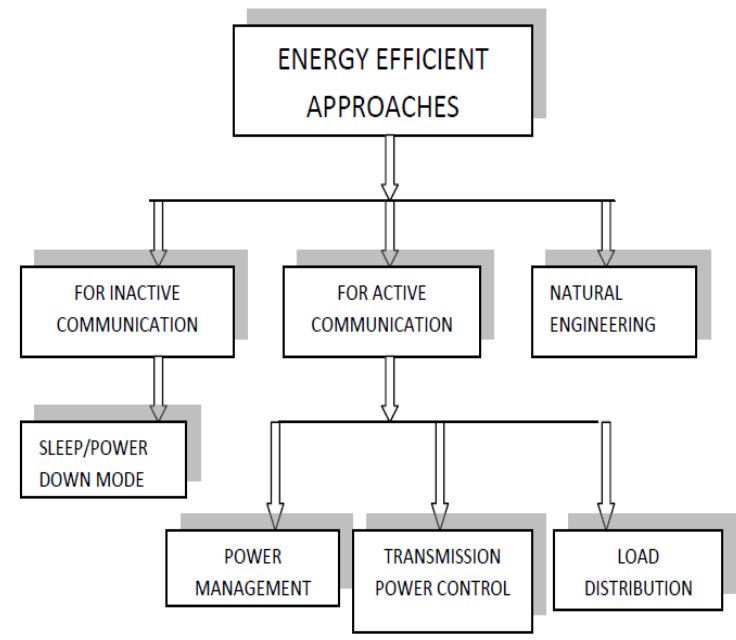

Figure 1. Division of energy minimizing processes

\subsection{Power Management Approach}

One way to combat the problem of energy deficiency is to minimize the power consumed by mobile devices. The idea [12] relies on the concept of triggering the nodes from high power mode to low power, which eventually leads to saving of energy. For efficient power management mechanism following spheres need to be considered:

- Which nodes will participate for power management process?

When to switch an active node to its sleeping mode?

The duration of an inactive node to remain dormant.

Minimum delay criteria between source and destination needs to be fulfilled in comparison with all nodes active.

The algorithm used for power management needs to follow distributed fashion.

As an idle node in active mode consumes same amount of energy as an active transmitter, therefore it should be necessary that algorithms allows as many nodes as possible to switch off their radio receiver.

\subsection{Transmission Power Control (TPC)}

TPC is one of the well known approaches used to save energy for wireless Ad-hoc networks. Reference [14] defines TPC by taking up a transmitter node $\mathrm{X}$. It is stated that for a given data transmission rate $\mathrm{R}$, the node $\mathrm{X}$ keeps its transmission power to a receiving node $\mathrm{Y}$ as low as needed for satisfying a target bit error rate $\mathrm{E}$ at the receiving node. Hence, by reducing the transmission power of nodes can lead to increase in network capacity.

The transmission power adjustment helps in maintaining a connected topology using the minimal power. So, the routing protocols based on controlling the transmission power are eligible to find best route which minimizes the total power between source and destination pair. Here transmitting power of mobile nodes [12] is adjusted in accordance with: signalto-interference and noise ratio (SINR) of the transmitting or receiving nodes, and distance between transmitter and receiver.

\subsection{Load Distribution}

Balancing the usage of energy in each node by choosing a path with underutilized nodes rather than shortest route is the main objective of this approach [12]. Although, it leads to longer routes, but routes are energy conserving as energy rich intermediate nodes are used. This process can seen more of load shedding task as it do not necessarily provide lowest energy path, but gives relief to overburdened nodes which happens to make longer network lifetime.

\subsection{Sleep/power Mode Approach}

This division focuses on inactive time of communication. With the emergence of low power states supported by hardware, it becomes essential to utilize them to save energy. Specifically in scenarios like MANETs, where energy as a resource has always been deficient.

In sleep mode [12], all nodes get inactive and packets cannot be delivered to a destination node. To overcome this problem, a master node is introduced. So, this node can coordinate the communication between the remaining slave nodes. Environments where the network is big such as multi hop MANET, more than one master node has to be introduced for convenience. This concept emerge out to be a master-slave network architecture, where except the master nodes, all nodes switch to energy saving mode i.e. sleep mode. Periodically these slave nodes get active to check out for any message from master node. If the master node is idle, they again switch to sleep mode.

\subsection{Natural Engineering}

From years researchers are working on nature inspired routing protocols which have eliminated several problems in the area such as battery life, scalability, maintainability, survivability, adaptability and so on[11]. Nature gifted solutions through ants, bees, wasps and birds have contributed in building up energy efficient algorithms. Bee ad-Hoc algorithm [15] and Energy Aware Ant-based Routing Algorithm (EAAR) [16] are algorithms based on the foraging behavior of insects to lessen the energy consumption.

Accordingly, algorithms have gone through different energy conserving strategies. One of which was Minimum Battery Cost routing algorithm (MBCR), the paths having nodes with less remaining battery capacity are not selected [14]. Problem was that individual battery capacity of a node got neglected while choosing the path having more remaining energy on average. This was rectified by Min-Max Battery cost routing (MMBCR) [17], as it selected the route according to battery capacity of all individual nodes. But this had its own discrepancies. As it did not ensure minimum transmission power, it reduced the network lifetime.

To the best of literature available, Min power consumption and max lifetime algorithm [8] is the latest one where battery of each host was used more fairly than the previous schemes. Eventually, it resulted in reducing the end to end delay and optimizing packet delivery ratio. Due to which this algorithm is taken into consideration for exploring its effect on protocols, as the research in MANETs should expand to match the rapid evolution of latest advancements in wireless communication technologies.

For this OLSR protocol which in itself is energy aware protocol has been taken several times for enhancing its energy metric. In [16], energy efficient optimized link state routing protocol (EE-OLSR) was introduced which modified the MPR selection mechanism based on willingness concept. It 
was noticed that EE-OLSR outperformed traditional OLSR in terms of throughput and average nodes lifetime.

Also, a metric based evaluation of OLSR proved to be more energy efficient in [18].The author focused on energy level as Quality of service (Qos) metric, which has been used for routing decisions in many energy-efficient routing protocol proposals.

\section{PROPOSED WORK}

The overall aim of this work is to design novel energy efficient OLSR protocol for mobile Ad hoc network. This had to accomplish by taking up proposed algorithm [8] known as Min power consumption and Max lifetime to be implemented over OLSR mechanism.

In this, a threshold value was taken up before simulation which indicated which node should participate in path discovery for a packet. The node having energy less than the threshold got destroyed from the path to be traversed and optimum path was followed. Adding to it, equal lengths of packets were transmitted so as to attain equal power consumption.

According to the algorithm design two things need to be taken care of:

- OLSR should decide the minimum power consumption in advance. This had to be done by considering the threshold value, and comparing it with nodal energy of each node participating. The one found lower than the value should be removed off from the path.

- For equal power consumption of nodes message with equal packet length should be transmitted. OLSR periodically assessed the residual energy of each node in order to make comparison with threshold.

\subsection{Algorithm Used}

The algorithm has been discussed precisely in the previous section. According to the algorithm, at the source node, divide the message into equal length of packets and select a node $\mathrm{i}$ where, Min $\left(\mathrm{E}_{\mathrm{i}}>\mathrm{Th}_{\mathrm{i}}\right)$ from all neighbouring node (here $\mathrm{E}_{\mathrm{i}}$ is the residual energy of node $\mathrm{i}$ and $\mathrm{Th}_{\mathrm{i}}$ is the fixed threshold according the algorithm).

Establish a route to destination wherein the energy level of all nodes is greater than its threshold value. Repeat the following steps in periodical interval t. Calculate the residual energy of each node in the route with the eq:

$\mathrm{E}_{\text {Res }}=\mathrm{E}-\mathrm{E}_{\mathrm{c}(\mathrm{t})}$

Where $\mathrm{E}$ is the initial energy of node,

$\mathrm{E}_{\mathrm{c}(\mathrm{t}) \text {, }}$ is the energy consumed during the time $\mathrm{t}$

$\mathrm{E}_{\mathrm{Res}}$ is the residual energy.

If $\mathrm{E}_{\mathrm{Res}}>$ threshold value continue the transmission through that node. Else dynamically find an alternate route for further transmission which satisfies the constraint.

\subsection{Simulation Scenario}

For implementing the proposed routing scheme we simulated dense wireless network, with 50 nodes moving in $1000 \times$ $1000 \mathrm{~m}$ area. Each node moves randomly in this area for 20 ms simulation time. The traffic sources used in the simulations generated constant bit rate (CBR) data traffic. Taking care of the equal packet size transmission, we have taken 512 bytes packet length.

\subsection{Energy Model}

Energy Model was setup as per the configuration shown in Table 1 over OLSR. According to it the initial, transmitting, receiving, idle and sleep power are taken.

Table 1. Energy Model Metrics

\begin{tabular}{|l|l|}
\hline \multicolumn{2}{|l|}{ ENERGY MODEL } \\
\hline Initial Power & 3.4 Joules \\
\hline Transmitting Power & 0.33 Joules \\
\hline Receiving Power & 1.0 Joules \\
\hline Idle power & 0.05 Joules \\
\hline Sleep power & 0.03 Joules \\
\hline
\end{tabular}




\section{SIMULATION RESULTS}

To evaluate ET-OLSR protocol, we used the NS-2 network simulator. Imperative parameters like packet delivery ratio, energy consumption, and throughput were considered for analysis. The threshold value was fixed to 1.8235888 joules. Henceforth the nodes having energy below it were automatically destroyed as shown in Figure 2, where Node: 0 having energy 1.734060 got eliminated.

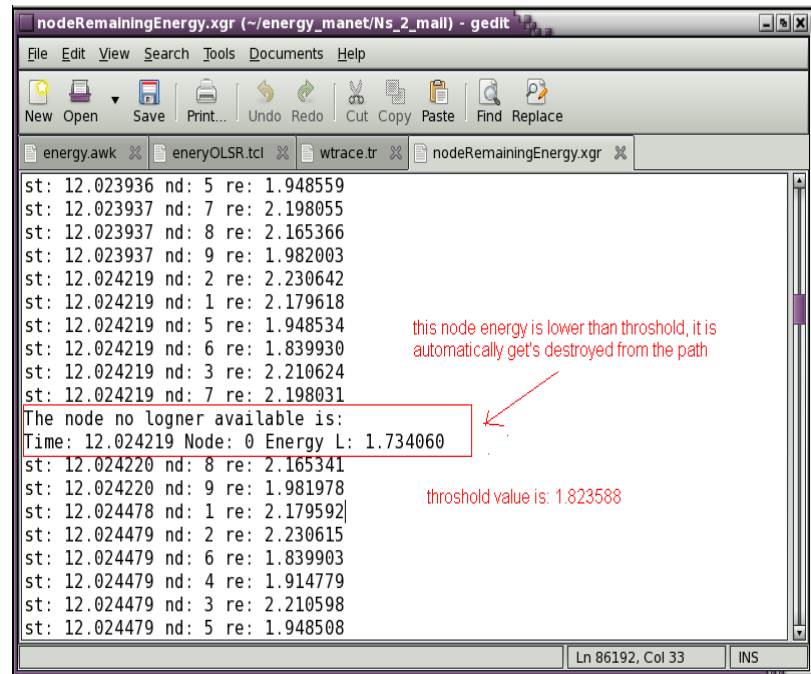

Figure 2. Working of the algorithm by taking up threshold

NS-2 simulator allows extracting many interesting parameters from simulation. We used this data to evaluate the performance of both traditional OLSR and ET-OLSR from energetic point of view. Figure 3 illustrates the packet delivery ratio (PDR) for both the protocols.PDR of both protocols shoot up in first 5 seconds. Afterwards the old OLSR lowers down. Both protocols show variations in the remaining seconds. The proposed ET-OLSR outshines from traditional in PDR statistics.

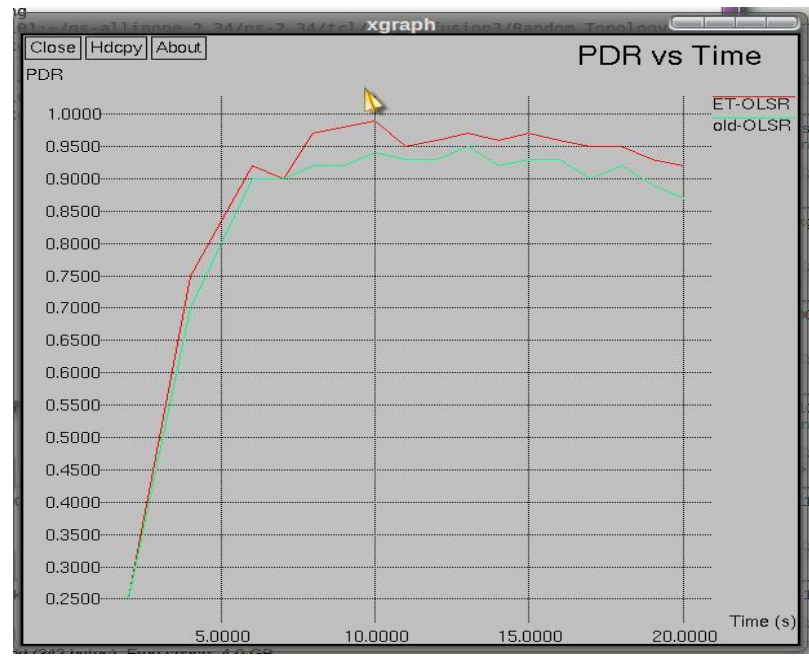

Figure 3. Packet delivery ratio comparison graph

As critical packets scenario was taken, the reliability of the routing increased. Due to which the increase in PDR was experienced in ET-OLSR.

Energy aware routing was the central issue of the implementation, hence energy consumption parameter was considered. Figure 4 displays the results for energy consumption trend followed by ET-OLSR (taken up as energy graph) and old OLSR (taken up as old energy). The graph proves that the energy consumed by traditional OLSR is more as compared to the newly implemented protocol.

According to the algorithm implemented over traditional OLSR, it was expected that the network lifetime will show improvement in the protocol. Eventually, the energy consumption of ET-OLSR is more successfully achieved.

In contrary, threshold parameter degraded in new ET-OLSR. The throughput of the protocols can be seen in Figure 5, where the new ET-OLSR clearly fails to exhibit better results. In the first 20 seconds both protocols peak up to the highest value, but at 20th second new ET-OLSR dips and remains constant for the simulation.

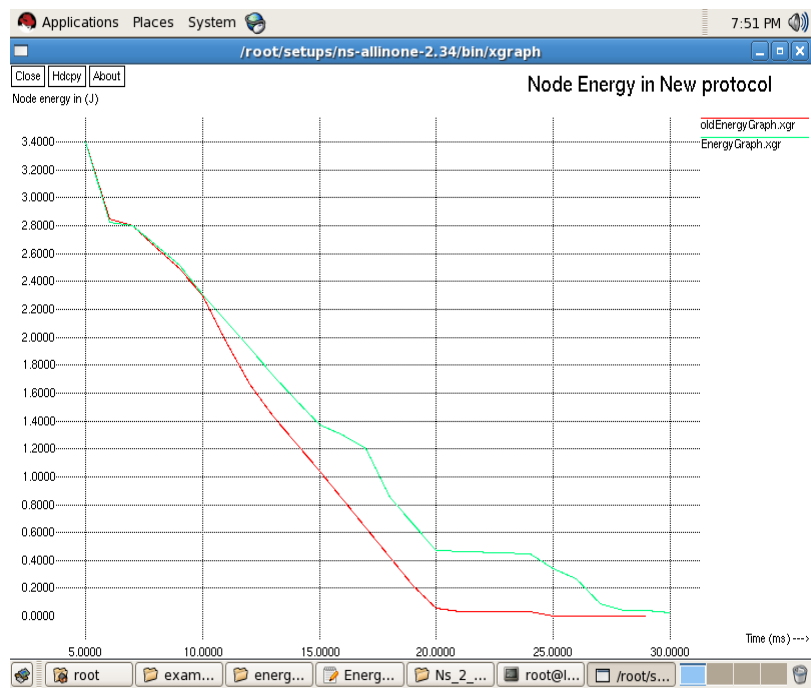

Figure 4. Energy consumption comparison graph

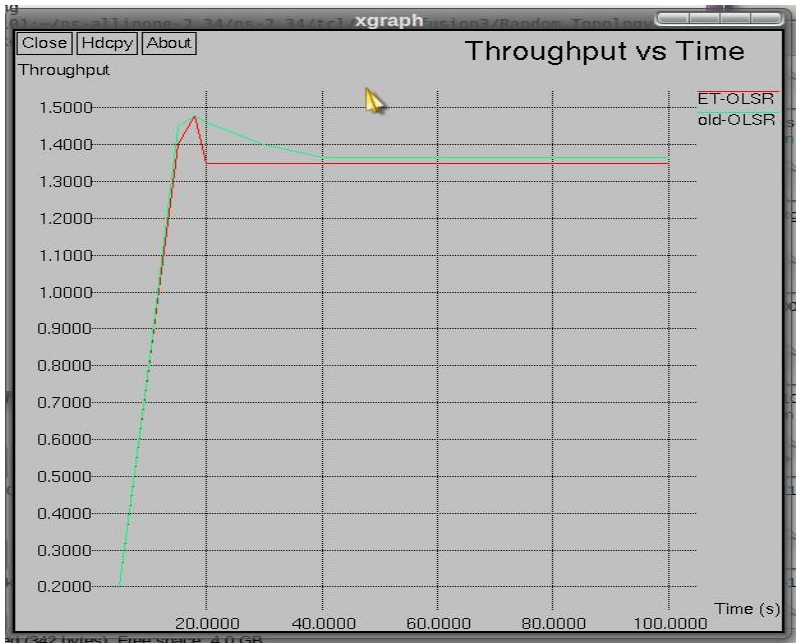

Figure 5. Throughput comparison graph

The results of new energy based threshold OLSR were compared with the traditional OLSR and it was found that proposed OLSR is more efficient from the traditional in energy, network lifetime and Packet delivery ratio, whereas throughput is better in the traditional one.

The average value of each parameter was taken for both protocols to get a clear picture of the results. The values of the simulated results are shown in Table 2 . 
Table 2. Comparison table of ET-OLSR and OLSR

\begin{tabular}{|l|l|l|}
\hline \multicolumn{1}{|c|}{ PARAMETERS } & \multicolumn{1}{|c|}{ ET-OLSR } & \multicolumn{1}{c|}{ OLSR } \\
\hline Energy Consumption & 2.344 joules & 3.48415 joules \\
\hline $\begin{array}{l}\text { Packet Delivery } \\
\text { Ratio }\end{array}$ & $87.96 \%$ & $85.63 \%$ \\
\hline Throughput & $1302.7 \mathrm{kbps}$ & $1339.23 \mathrm{kbps}$ \\
\hline
\end{tabular}

\section{CONCLUSION}

In this work, power management algorithm named Minimum Power Consumption and Maximum Lifetime Algorithm was considered over OLSR, where threshold value for energy was taken as 1.8235888 joules. We proposed a new protocol ETOLSR taking energy enhancement as central issue of concern. From comparison graphs we implied that ET-OLSR outperforms the traditional OLSR in terms of PDR and energy consumption. As it could not do the same for throughput, it is suitable for environments where the parameter can be ignored.

In future, critical packet optimization of throughput can be considered. The security parameter can be implemented on ET-OLSR, to make it more robust.

\section{REFERENCES}

[1] Ramkumar KR,Ravichandran CS, "Analog Based Routing Algorithm using Ant agents for MANETs", International Conference on Modeling Optimization and Computing,Procedia Engineering 38 ( 2012 ) 3139 3151

[2] AL-Gabri Malek et al., "Improved the Energy of Ad Hoc On-Demand Distance Vector Routing Protocol", IERI Procedia 2 ( 2012 ) $355-361$.

[3] C. Jones, K. Sivalingam, P. Agarwal, J.C. Chen, "A survey of energy efficient network protocols for wireless and mobile networks", ACM/Kluwer Wireless Networks 7 (4) (2001) 343-358.

[4] A. Campbell, M. Conti, S. Giordano, "Special issue on mobile ad hoc network", ACM/Kluwer MONET 8 (5) (2003).

[5] K. Arulanandam, Dr. B. Parthasarathy, "A New Energy Level Efficiency Issues in MANET", International Journal of Reviews in Computing 2009.

[6] C.F. Chiasserini, I. Chlamtac, P. Monti, A. Nucci, "Optimal energy design of wireless ad hoc networks", in:
E. Gregori, M. Conti, A.T. Campbell, G. Omidyar, M. Zukerman (Eds.), Lecture Notes in Computer Science, vol. 2345, Springer, Berlin, 2002.

[7] C. Petrioli, R. Rao, J. Redi, "Special Issue on Energy Conserving Protocols", ACM/Kluwer MONET 6 (3) (2001).

[8] S. Suganya and S. Palaniammal, "An Optimized energy Consumption Algorithm for MANETS", Procedia Engineering 38 ( 2012 ) $903-910$.

[9] RFC 3626, IETF organisation.

[10] Shipra Gautam, Rakesh Kumar, "A Review of EnergyAware Routing Protocols in MANETs", International Journal of Modern Engineering Research (IJMER), Vol.2, Issue.3, May-June 2012 pp-1129-1133.

[11] Neeraj Tantubay , Dinesh Ratan Gautam ${ }^{2}$ and Mukesh Kumar Dhariwal , "A Review of Power Conservation in Wireless Mobile Adhoc Network (MANET)", IJCSI International Journal of Computer Science Issues, Vol. 8, Issue 4, No 1, July 2011.

[12] Chansu Yu, ben Lee, Hee Yong Yum, "Energy Efficient Routing Protocols for Mobile Ad Hoc Networks", research supported in part by Cleveland State University, EFFRD Grant No. 0120-0630-10.

[13] S. Misra et al., "An ant swarm-inspired energy-aware routing protocol for wireless ad-hoc networks" The Journal of Systems and Software 83 (2010) 2188-2199.

[14] S. Lindsey, K. Sivalingam, C. S. Raghavendra, "Power Optimization in Routing Protocols for Wireless and Mobile Networks," Handbook of Wireless Networks and Mobile Computing, I. Stojmenovic, Ed., Wiley, 2001.

[15] Horst. F. Wedde, Muddassar Farooq, Thorsten Pannenbaecker, Bjoern Vogel,Christian Mueller, Johannes Meth and Rene Jeruschkat, "BeeAdHoc: An Energy Efficient Routing Algorithm for Mobile Ad Hoc Networks Inspired by Bee Behavior", GECCO'05, June 25-29, 2005, Washington, DC, USA.Copyright 2005

[16] S. Misra et al., "An ant swarm-inspired energy-aware routing protocol for wireless ad-hoc networks" The Journal of Systems and Software 83 (2010) 2188-2199.

[17] C.-K. Toh "Maximum Battery Life Routing to Support Ubiquitous Mobile Computing in Wireless Ad Hoc Networks", in IEEE Communication Magazine, June 2001.

[18] T. Kunz, R. Alhalimi , "Energy-efficient proactive routing in MANET: Energy metrics accuracy" , Ad Hoc Networks 8 (2010) 755-766. 